\title{
A Single-Blinded Trial Using Resting-State Functional Magnetic Resonance Imaging of Brain Activity in Patients with Type 2 Diabetes and Painful Neuropathy
}

\author{
Qing Zhang · Peng Zhang · Rui Yan · Xianghong Xu • \\ Cunnan Mao · Xiaomei Liu · Fengfei Li · Jianhua Ma • \\ Lei Ye $\cdot$ Zhijian Yao $\cdot$ Jindan $\mathrm{Wu}$
}

Received: October 10, 2018 / Published online: November 30, 2018

(C) The Author(s) 2018

\section{ABSTRACT}

\begin{abstract}
About two-thirds of patients with painful diabetic neuropathy (PDN) suffer from anxiety and/or depression disorders. However, the pathogenesis of PDN is unclear, in particular with respect to the mechanism associated with
\end{abstract}

Enhanced digital features To view enhanced digital features for this article go to https://doi.org/10.6084/ m9.figshare.7308761.

Qing Zhang and Peng Zhang contributed equally.

Q. Zhang · P. Zhang - X. Xu - X. Liu - F. Li · J. Ma · J. Wu $(\bowtie)$

Department of Endocrinology, Nanjing First

Hospital, Nanjing Medical University, Nanjing,

China

e-mail: wujindandan@sina.com

Q. Zhang

Department of Endocrinology, The Affiliated

Jiangning Hospital of Nanjing Medical University,

Nanjing, China

R. Yan $\cdot$ Z. Yao $(\bowtie)$

Department of Psychiatry, Nanjing Brain Hospital, Nanjing Medical University, Nanjing 210029, China e-mail: zjyao@njmu.edu.cn

L. Ye

National Heart Research Institute Singapore,

National Heart Centre Singapore, Singapore,

Singapore

C. Mao

Department of Radiology, Nanjing First Hospital,

Nanjing Medical University, Nanjing, China the central nervous system. We used the neuroimaging techniques of fraction amplitude of low-frequency fluctuation (fALFF) and regional homogeneity of resting-state functional magnetic resonance imaging (fMRI) to explore the brain activity in patients with PDN. The symptoms, signs and mental conditions of 19 patients with PDN and of 18 patients with nonpain neuropathy were assessed separately and compared. Blood oxygenation level-dependent resting-state fMRI scans of the brain were performed in all 37 patients with neuropathy and in 15 gender- and age-matched healthy controls. Our data showed that patients with PDN had increased insulin resistance $(p=0.03)$, increased depression $(p=0.02)$ and increased anxiety $(p<0.001)$ compared with the controls and that all of these conditions were associated with abnormal spontaneous activities in several regions of the brain, including the somatosensory, cognitive and emotional regions. The duration of diabetes, level of glycated hemoglobin, homeostasis model assessment of insulin resistance and estimated glomerular filtration rate were significantly correlated to abnormal spontaneous activity in patients' brains. These results lead to the conclusion that patients with PDN have abnormal brain activity, indicating that the central nervous system may contribute to painful diabetic neuropathy. Trial registration: ClinicalTrials.gov identifier NCT03700502. 
Keywords: Fraction amplitude of lowfrequency fluctuation; Painful diabetic neuropathy; Resting state functional MRI; Regional homogeneity

\section{INTRODUCTION}

Neuropathy is one of the more common chronic complications of diabetes, with about $60 \%$ of patients with type 2 diabetes (T2D) having varying degrees of peripheral neuropathy, among whom one-third have neuralgia [1]. T2D patients with neuralgia suffer from pain at night, which in turn affects sleep [2]. Nearly two-thirds of patients with painful diabetic neuropathy (PDN) suffer from anxiety and/or depression comorbidities, which contribute to a poorer life quality of patients with PDN [3]. However, the pathogenesis underlining PDN is still unclear [4]. The Position Statement on diabetic neuropathy issued by the American Diabetes Association in 2017 recommends the use of psychotropic drugs, such as duloxetine and pregabalin, as first-line medication for the treatment of PDN pain [5]. Studies focusing on the central mechanism of PDN are currently attracting great interest, with some authors suggesting that the central nervous system may be involved in the pain [3]. One recent study showed that in patients with PDN there was a reduced volume of gray matter around the somatosensory cortex and an increasing blood flow in the thalamus [6]. Patients with PDN have also been shown to have thalamic and limbic system nerve dysfunction, as well as destruction of the default network and attention network [3].

Resting-state functional magnetic resonance imaging (rs-fMRI) can reflect the degree and synchronicity of brain neuron activity in a specific area of the brain and has been widely used in studies of central nervous system [7]. Fraction amplitude of low-frequency fluctuation (fALFF) and regional homogeneity (ReHo) are two common fMRI technologies that are used in central nervous system research [8]. We performed a single-blinded clinical trial in which rs-fMRI single analysis (fALFF and ReHo) were employed to characterize spontaneous brain activity in patients with T2DM and painful or non-painful neuropathy. The aim of our study was to find correlations between brain activities and clinical indicators or neuropsychiatric scale scores that might provide new clues for the diagnosis and treatment of PDN.

\section{METHODS}

\section{Participants and trial description}

Patients with T2D and painful (Group-PDN) or non-painful (Group-N) neuropathy were recruited to the study from the Department of Endocrinology of the Nanjing First Hospital, between 2016 and 2017. Prior to entry to the study, each patient was given a complete description of the study and provided written informed consent.

\section{Inclusion Criteria for Patients}

The inclusion criteria for patients were: (1) voluntary participation and signing of informed consent form; (2) age 18-60 years, with junior high school education or higher (3) met the 1999 World Health Organization T2D diagnostic criteria; (4) diagnosis of $\mathrm{PN}$ based on the following three items, of which patients with painful neuropathy have symptoms of pain: (1) neuropathy occurred after diagnosis of diabetes; (2) patient had clinical manifestations of pain, numbness and abnormal sensation; (3) at least one abnormal finding in the following five examinations: ankle reflex, vibratory sensation, pressure sensation, temperature sensation, and acupuncture pain.

\section{Inclusion Criteria for Healthy Controls}

The inclusion criteria for healthy controls were: (1) voluntary participation and signing of informed consent; (2) age 18-60 years, with junior high school education or higher; (3) no history of diabetes in the past and glycated hemoglobin (HbA1c) level of between 4 and $6 \%$; (4) normal results on the anxiety and depression scales. 


\section{Exclusion Criteria for all Subjects}

The exclusion criteria for all participants were: (1) left-handedness; (2) neuropathy caused by other causes, such as cervical spondylosis, cerebral infarction, Guillain-Barré syndrome, severe arteriovenous disease and neurotoxic changes caused by drugs and renal insufficiency; (3) presence of severe cerebral vascular disease; (4) diagnosed mental disorders, such as depression, anxiety and Alzheimer's disease; (5) intolerance to MRI of the head due to, for example, the presence of metal (stents, metal dentures, internal fixation plates, among others) and claustrophobia.

The study was approved by the medical ethics committee of the first hospital of Nanjing, Nanjing, China (Document Number: KY20180205-01). All procedures performed in studies involving human participants were in accordance with the Institutional Review Board committee of the first hospital of Nanjing and with the 1964 Helsinki declaration and its later amendments or comparable ethical standards.

\section{Patient Clinical Data}

The baseline data, nerve and mental scale scores and results of the fundus examination, electrophysiological examination, biochemical examination (fasting blood glucose, fasting $\mathrm{C}$ peptide, HbAc1 level, blood lipid, creatinine and urine albumin) were recorded for all patients. Body mass index (BMI) was calculated as body weight $(\mathrm{kg}) /$ height $^{2}\left(\mathrm{~m}^{2}\right)$; homeostasis model assessment-insulin resistance (HOMA-IR) was calculated as 1.5 + fasting blood glucose $(\mathrm{mmol} / \mathrm{L}) \times$ fasting $\mathrm{C}$ peptide $(\mathrm{ng} / \mathrm{mL}) /(2.8 \times$ 0.333) [9]; homeostasis model assessment- $\beta$ (HOMA- $\beta$ ) was calculated as $270 \times$ fasting $C$ peptide $(\mathrm{ng} / \mathrm{mL}) /[0.333 \times$ (fasting blood glucose $(\mathrm{mmol} / \mathrm{L})-3.5)]$ [9].

\section{Nerve and Mental Scale Assessment}

Nerve and mental scales were assessed independently by three trained doctors based on the symptoms, signs and mental conditions of the patients. These include pain symptoms and neurologic signs using the visual analogue scale
(VAS), Toronto Clinical Scoring System (TCSS) and Leeds Assessment of Neuropathic Symptoms and Signs (LANSS); (2) mental conditions using the Self-Rating Anxiety Scale (SAS), Selfrating Depression Scale (SDS), Hamilton Anxiety Scale (HAMA), Hamilton Depression Scale (HAMD) and social function scale.

\section{MRI Scanning Techniques}

The whole blood oxygenation-level Dependent (BOLD) signal was collected using a Philips Ingenia 3.0T MRI scanner (Philips BV, Amsterdam, The Netherlands).

\section{T1-Weighted Three-Dimensional Structure of the Whole Brain}

For the T1-weighted three-dimensional (T1WI3D) brain imaging, a T1-FLAIR sequence was acquired. The scanning parameters were: parallel to the front and rear joint lines; 170 axes. Pulse repetition time (TR)/echo time (TE) $8.2 / 3.8 \mathrm{~ms}$; visual field (field of view [FOV]) $240 \times 240 \mathrm{~mm}$; matrix $240 \times 222$; layer thickness $1 \mathrm{~mm}$; scanning time $5^{\prime} 29^{\prime \prime}$. For those patients with no brain structure abnormalities, a rs-fMRI scan was performed.

\section{Resting State-fMRI}

For the rs-fMRI, the imaging technique used was gradient-recalled echo-planar imaging (GRE$\mathrm{EPI})$. The scanning parameters were TR/ TE $2000 / 30 \mathrm{~ms}$; FOV $220 \times 220 \mathrm{~mm}$; matrix 72 $\times$ 70; 36 layers; layer thickness $4 \mathrm{~mm}$; scanning time $12^{\prime} 45^{\prime \prime}$. During the scan, subjects closed their eyes on the examination bed but remained awake; they were asked to remain still, in particular to keep the head fixed, and to avoid deliberate mental activities.

\section{Data Processing and Statistical Analysis of Acquired Imaging Sequences}

Processing of rs-fMRI Data We used the REsting-State fMRI data analysis Toolkit (REST) and DPARSF 2.2 plug-in software to analyze rsfMRI data on the Matlab R2012b platform, carrying out the steps in the order listed as follows:

1. Remove the first 10 time points to eliminate early detection interference. 
2. Run MRIcron software to transform the remaining 220 time points in the data format.

3. Use the middle layer (35th level) as the reference layer for time correction.

4. Calibrate head movements with translational $2 \mathrm{~mm}$ and angle $2^{\circ}$, and eliminate inconsistent data.

5. Normalize image space to Montreal standard head anatomic template and resample by $3 \times 3 \times 3 \mathrm{~mm}$ in size.

6. Use the Gauss kernel function of $4 \mathrm{~mm}$ with full width and half height for spatial smoothing (this step is not required in ReHo).

7. Delete the linear trend caused by too long image acquisition time.

8. Filter at a frequency of $0.01-0.08 \mathrm{~Hz}$.

Extraction of fractional amplitude of low-frequency fluctuation Data The first step in extracting the fractional amplitude of low-frequency fluctuation (fALFF) data was to select the whole brain as the seed in order to calculate the ALFF value of each voxel. The fALFF value was obtained by dividing the total value of the 0 - to $0.25-\mathrm{Hz}$ amplitude of the whole frequency band. The average amplitude of the whole brain was then divided, and the standard deviation was subtracted. Standard values were compared to determine differences across each group. The second step was to analyzed the fALFF data of each group using one-way analysis of variance (ANOVA). On the basis of differences between the three groups, we carried out two-independent samples $t$ tests between the PDN- and $\mathrm{N}$-groups and performed the Alphasim multiple comparison correction using Data Processing Assistant for Resting-State fMRI (DPARSF) version 2.2. We used these parameters as the significance threshold, which was combined with the individual voxel at a significance level of $p<0.05$ and a minimum cluster size of $459 \mathrm{~mm}^{3}$. We obtained the parameter by using the cluster threshold size estimator plug-in in REST software. In applying the parameter, the family-wise (corrected) false probability rate was set at $p<0.05$. The automated anatomical labeling (AAL) function was employed to partition the brain and the Slice viewer of REST was used to determine the Montreal Neurological Institute (MNI) coordinates of the peak points, which were defined as statistically significant regions.

Extraction of ReHo Data The first step in extracting ReHo data was to use the Kendall coefficient of concordance (KCC) to calculate the similarity in time series of 27 adjacent individual voxels. The smReHo graphs obtained from the standardized ReHo diagram, proceeded by $4 \times 4 \times 4$ - $\mathrm{mm}^{3}$ Gauss smoothing, were used for the statistical analysis. The second step was to analyze the ReHo data of the three groups using ANOVA. Based on the different brain regions of the three groups, the two-independent samples $t$ test was conducted between Group-PDN and group-N. After performing the Alphasim multiple comparison correction, obtaining the threshold of the single activator at $p<0.01$, the minimum cluster size of $91 \mathrm{~mm}^{3}$ was defined as a region with statistically significant difference. MNI coordinates, voxels and peak values of different brain regions were obtained by REST.

\section{Statistical Analysis of Clinical Data}

SPSS version 22.0 statistical software (IBM Corp., Armonk, NY, USA) was used to analyze each patient's clinical index and nerve mental scale. Data which were normally distributed data are reported as the mean \pm standard deviation, and the normal and variance homogeneity tests were subsequently carried out. The comparison of the mean values between groups was tested by the independent-samples $t$ test. Data which were not normally distributed were analyzed by logarithmic transformation. Enumeration data are expressed as frequency. The Chi-square test was used to compare groups. Statistical significance was set at $p<0.05$.

REST software was used to extract fALFF and ReHo values for seed points with a significant difference in brain region coordinates, so that the volume of voxel was quantified to facilitate Pearson correlation analysis with clinical indicators and neuropsychiatric scales. Statistical significance was set at $p<0.05$. 


\section{RESULTS}

\section{Patient Clinical Data}

We consecutively recruited 56 right-handed patients with T2D. Of these, 20 (12 males, 8 females) were assessed to have painful neuropathy and allotted to Group-PDN; 20 (13 males, 7 females) were assessed to have nonpainful neuropathy and allotted to Group-N. A third group of 16 healthy volunteers (9 males, 7 females), matched for age, sex, and years of education, was also included as the control group. One patient in Group-PDN, two patients in Group- $\mathrm{N}$ and one healthy volunteer were subsequently excluded due to head motion of $>2 \mathrm{~mm}$ during the MRI scans. There was no significant difference in gender, age and education level after the clinical data of three groups were tested by normal distribution and homogeneity of variance. There was no significant difference in the course of disease between Group-PDN and Group-N (Table 1).

The HOMA-IR, TCSS, SAS, HAMD, HAMA and social function scale scores were all significantly different between Group-PDN and Group-N patients $(p<0.05)$, while age, sex, waist-to-hip ratio, hemoglobin, HbA1c, C-peptide, blood lipid, BMI, creatinine, urine protein quantitative, estimated glomerular filtration rate (eGFR), HOMA- $\beta$, neurophysiology and retinopathy were not significantly different $(p>0.05$; Table 2$)$.

\section{Results of the fALFF}

One-way ANOVA showed that the fALFF values of the three groups differed significantly in the Cerebelum_9_R, Fusiform_L, Temporal_Pole_ Sup_R, Occipital_Mid_R, Frontal_Inf_Orb_L, Occipital_Mid_L, Temporal_Mid_R, Cingulum_ Post_R, Postcentral_R, and Supp_Motor_Area_R sequences $(p<0.05$; Alphasim correction: $p$ $<0.05$; minimum cluster size $459 \mathrm{~mm}^{3}$; Fig. 1).

Compared with Group-N, the fALFF values of Group-PDN in the Temporal_Inf_L, Lingual_R, Occipital_Mid_L, Occipital_Sup_L, Postcentral_L and Parietal_Inf_L sequences were significantly higher, while the fALFF values in the Temporal_Pole_Sup_L, Frontal_Inf_Oper_L, Temporal_Sup_R, Rolandic_Oper_L, Cingulum_Post_L, Precentral_L, Supp_Motor_Area_R and Supp_Motor_Area_L sequences decreased significantly $(p<0.05$; Alphasim correction: $p$ $<0.05$; minimum cluster size $459 \mathrm{~mm}^{3}$; Fig. 2).

\section{Results of ReHo}

One-way ANOVA analysis showed that the ReHo values indicated significant differences in brain activity, including in the temporal lobe, frontal lobe, occipital lobe and cerebellum, among the three groups ( $p<0.05$; Fig. 3$)$.

The ReHo values of Group-PDN in the Frontal_Mid_R, Parietal_Inf_L sequence were significantly higher than those of Group-N, while those in the Heschl_L sequence were significantly lower ( $p<0.05$; Fig. 4 ).

Table 1 Demographic data for the patients in the three study groups included in the analysis

\begin{tabular}{lllll}
\hline Data & Group-PDN $(\boldsymbol{n}=\mathbf{1 9})$ & Group-N $(\boldsymbol{n}=\mathbf{1 8})$ & Control group $(\boldsymbol{n}=\mathbf{1 5})_{\boldsymbol{p} \text { value }^{\mathbf{a}}}$ \\
\hline Gender (male/female) & $12 / 7$ & $12 / 6$ & $8 / 7$ & 0.72 \\
Age (year) & $53.8 \pm 8.1$ & $54.1 \pm 6.9$ & $53.9 \pm 5.4$ & 0.88 \\
Education (year) & $9.9 \pm 3.3$ & $10.6 \pm 3.9$ & $11.4 \pm 2.3$ & 0.47 \\
Course of disease (number of years) & $8.9 \pm 5.4$ & $9.8 \pm 6.9$ & - & 0.93 \\
\hline
\end{tabular}

Data are presented as the mean \pm standard deviation $(\mathrm{SD})$

Group-PDN Patients with type 2 diabetes (T2D) and pain neuropathy; Group-N patients with T2D and non-pain neuropathy, Control group healthy volunteers

${ }^{a} p<0.05$ was considered to indicate a significant difference, according to one-way analysis of variance (ANOVA) 
Table 2 Demographic and clinical data for the patients of Group-PDN and Group-N

\begin{tabular}{|c|c|c|c|}
\hline Demographic and clinic data & Group-PDN $(n=19)$ & Group-N & $p$ value $^{\mathrm{a}}$ \\
\hline Age (year) & $53.8 \pm 8.1$ & $54.1 \pm 6.9$ & 0.82 \\
\hline Gender (male/female) & $12 / 7$ & $12 / 6$ & 0.82 \\
\hline Education (year) & $9.9 \pm 3.3(0-15)$ & $10.6 \pm 3.9(0-16)$ & 0.51 \\
\hline Weight $(\mathrm{kg})$ & $68.29 \pm 9.65(49-94)$ & $69.19 \pm 11.69(51-95)$ & 0.51 \\
\hline $\operatorname{BMI}\left(\mathrm{kg} / \mathrm{m}^{2}\right)$ & $24.84 \pm 3.50(18.67-29.94)$ & $24.46 \pm 2.31(20.43-30.08)$ & 0.70 \\
\hline Waist-to-hip ratio & $0.93 \pm 0.04(0.83-1.01)$ & $0.94 \pm 0.05(0.88-1.10)$ & 0.83 \\
\hline Hemoglobin $(\mathrm{g} / \mathrm{L})$ & $129.21 \pm 12.10(96-153)$ & $136.61 \pm 15.25(107-164)$ & 0.19 \\
\hline Cholesterol $(\mathrm{mmol} / \mathrm{L})$ & $4.95 \pm 1.17(2.50-7.06)$ & $5.15 \pm 1.29(3.28-7.53)$ & 0.62 \\
\hline Triglyceride $(\mathrm{mmol} / \mathrm{L})$ & $2.36 \pm 2.67(0.68-12.28)$ & $2.43 \pm 3.04(0.62-13.41)$ & 0.88 \\
\hline HbAlC (\%) & $8.45 \pm 1.93(6.0-12.8)$ & $8.68 \pm 1.73(5.2-11.5)$ & 0.71 \\
\hline C-peptide $(\mathrm{ng} / \mathrm{mL})$ & $1.61 \pm 0.93(0.45-3.65)$ & $1.13 \pm 0.66(0.2-2.55)$ & 0.08 \\
\hline Fasting blood glucose $(\mathrm{mmol} / \mathrm{L})$ & $8.97 \pm 3.13(4.4-15.04)$ & $7.59 \pm 3.55(3.75-19.16)$ & 0.22 \\
\hline HOMA-IR & $17.43 \pm 10.44(4.27-37.72)$ & $10.92 \pm 6.64(2.44-27.36)$ & $0.03^{*}$ \\
\hline HOMA- $\beta$ & $320.18 \pm 242.94(71.36-972.97)$ & $377.93 \pm 450.97(45.56-1988.05)$ & 0.88 \\
\hline Creatinine $(\mu \mathrm{mol} / \mathrm{L})$ & $67.98 \pm 19.15(36.1-117.5)$ & $69.24 \pm 32.03(42.2-183.5)$ & 0.33 \\
\hline Urine protein $(\mathrm{mg} / 24 \mathrm{~h})$ & $331.05 \pm 553.69(7.03-1860)$ & $205.11 \pm 382.19(4.16-1200)$ & 0.52 \\
\hline $\mathrm{eGFR}\left(\mathrm{ml} /\left(\mathrm{min} \times 1.73 \mathrm{~m}^{2}\right)\right.$ & $107.93 \pm 25.22(59.16-168.96)$ & $111.97 \pm 28.48(35.24-151.41)$ & 0.65 \\
\hline Neurophysiology (abnormal/normal) & $(15 / 1)$ & $(12 / 3)$ & 0.25 \\
\hline retinopathy (abnormal/normal) & $(9 / 5)$ & $(9 / 9)$ & 0.42 \\
\hline VAS & $6.8 \pm 1.9$ & - & - \\
\hline TCSS & $9.7 \pm 3.4$ & $6.2 \pm 1.8$ & $0.00^{*}$ \\
\hline LANSS & $17.4 \pm 4.5$ & - & - \\
\hline SDS & $35.2 \pm 7.1$ & $31.9 \pm 6.2$ & 0.14 \\
\hline SAS & $39.8 \pm 8.1$ & $28.8 \pm 4.4$ & $0.00^{*}$ \\
\hline HAMD & $9.0 \pm 7.2$ & $4.8 \pm 2.7$ & $0.02^{*}$ \\
\hline HAMA & $7.1 \pm 4.3$ & $3.8 \pm 2.2$ & $0.00^{*}$ \\
\hline Social function (work) & $5.2 \pm 2.7$ & $2.8 \pm 2.4$ & $0.01^{*}$ \\
\hline Social function (life) & $5.0 \pm 2.9$ & $2.3 \pm 2.0$ & $0.01^{*}$ \\
\hline
\end{tabular}


Table 2 continued

\begin{tabular}{llll}
\hline Demographic and clinic data & Group-PDN $(\boldsymbol{n}=\mathbf{1 9})$ & Group-N & $\boldsymbol{p}$ value $^{\mathbf{a}}$ \\
\hline Social function (family) & $5.3 \pm 3.1$ & $2.1 \pm 1.7$ & $0.00^{*}$ \\
\hline
\end{tabular}

Data are presented as the mean \pm SD with the range (minimum-maximum) given in parenthesis where appropriate $B M I$ Body mass index; HbAIC glycated hemoglobin; HOMA-IR homeostasis model assessment-insulin resistance; $H O M A$ $\beta$ homeostasis model assessment $\beta$; $e G F R$ estimated glomerular filtration rate; $V A S$ visual analogue scale; TCSS Toronto Clinical Scoring System; LANSS Leeds Assessment of Neuropathic Symptoms and Signs; SDS Self-rating Depression Scale; $S A S$ Self-Rating Anxiety Scale; HAMD Hamilton Depression Scale; HAMA Hamilton Anxiety Scale

${ }^{a} p<0.05$ was considered to indicate a significant difference $\left({ }^{*}\right)$ between groups, according to the independent-sample $t$ test

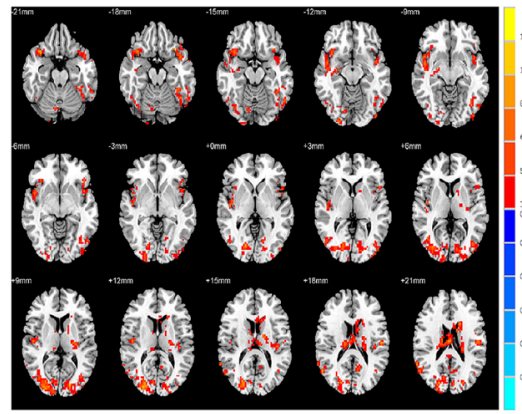

(a)

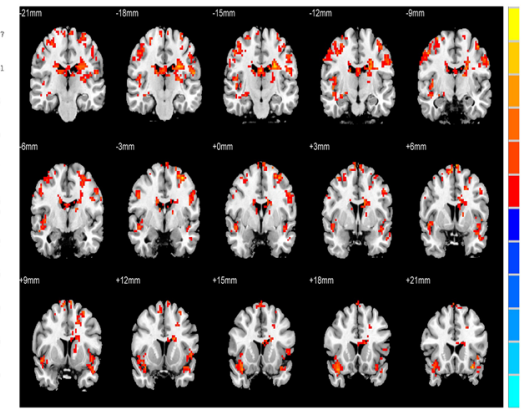

(b)

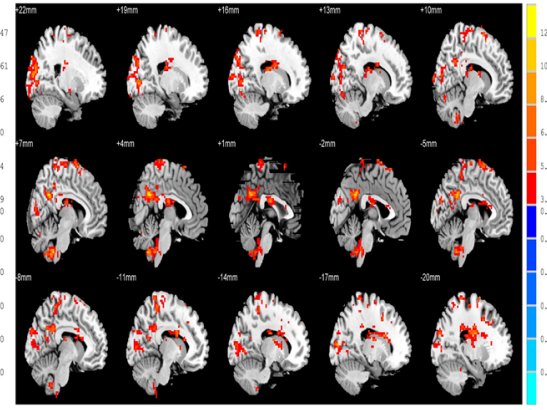

(c)
Fig. 1 Images of the fraction amplitude of low-frequency fluctuation (fALFF) in the transverse plane (a), coronal plane (b) and sagittal plane (c) to show areas that had significantly different activity intensity among the three

\section{Correlation Between fALFF and ReHo Values in Abnormal Brain Regions and Clinical Index in Groups-PDN and -N}

Correlation between the fALFF and ReHo values in abnormal brain regions and the clinical index in the Group-PDN and Group-N according to the Pearson correlation analysis indicated that the duration of diabetes, HbA1c level, HOMA-IR values and eGFR values were significantly correlated to the fALFF and ReHo values $(p<0.05$, respectively). We performed a binary logistic stepwise regression analysis, controlled for duration of diabetes, HbA1c, HOMA-IR and eGFR values, to identify which factors contributed to the fALFF and ReHo values. In Group-PDN, the fALFF value in the Fronta1_Inf_Oper_L sequence was negatively correlated with the eGFR value $(r=-0.49 ; p=0.03)$ and with HbA1c level $(r=-0.54 ; p=0.02)$, the groups. The fALFF data were analyzed by analysis of variance (ANOVA). Red color indicates an area that has significant difference in activity intensity

fALFF value in the Occipital_Mid_L sequence was negatively correlated with the HOMA-IR value $(r=-0.61 ; p=0.01)$ and the ReHo value in the Frontal_Mid_R sequence was positively correlated with the duration of diabetes $(r$ $=0.63 ; p=0.00$ ). In Group-N, the fALFF value in the Frontal_Inf_Oper_L sequence was positively correlated with the eGFR ( $r=0.52 ; p=0.03)$, the fALFF value in the Parietal_Inf_L sequence was positively correlated with HbA1c level $(r=0.53 ; p=0.02)$ and the value of fALFF in the Postcentral_L sequence was negatively correlated with the HOMA-IR value $(r=-0.56, p=0.01)$. There was no significant correlation between the fALFF value of the abnormal brain area and the anxiety and depression score, the duration of diabetes and HOMA- $\beta$ value $(p>0.05)$. There was also no significant correlation between the ReHo value of the abnormal brain area and the anxiety and 


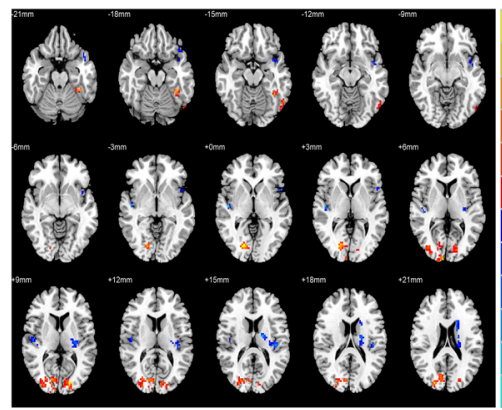

(a)

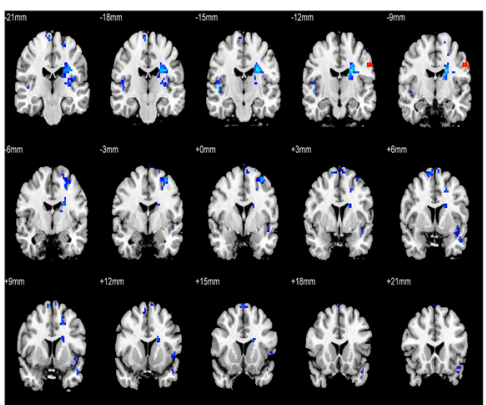

(b)

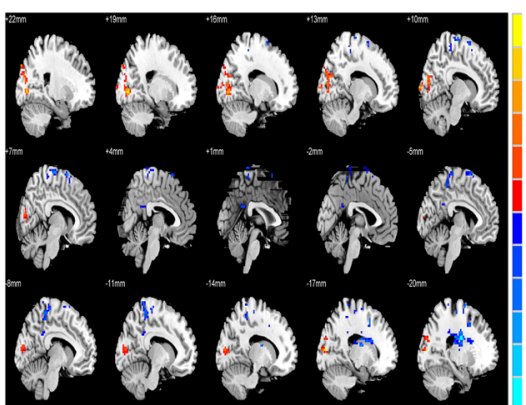

(c)

\begin{tabular}{|l|c|c|c|c|c|}
\hline \multirow{2}{*}{ aal } & \multicolumn{3}{|c|}{ Peak point } & K value & T value \\
\hline Temporal_Inf_L & $\mathbf{x}$ & $\mathbf{y}$ & $\mathbf{z}$ & & \\
\hline Temporal_Pole_Sup_L & -39 & -42 & -18 & 38 & 3.80 \\
\hline Frontal_Inf_Oper_L & -48 & 9 & -24 & 24 & -3.23 \\
\hline Temporal_Sup_R & -51 & 12 & 0 & 32 & -2.86 \\
\hline Lingual_R & 48 & -15 & 0 & 35 & -4.22 \\
\hline Occipital_Mid_L & 18 & -81 & 0 & 226 & 4.17 \\
\hline Rolandic_Oper_L & -18 & -87 & 9 & 51 & 4.00 \\
\hline Occipital_Sup_L, & -42 & -24 & 15 & 49 & -3.94 \\
\hline Cingulum_Post_L & -21 & -87 & 24 & 18 & 2.76 \\
\hline Postcentral_L & 0 & -51 & 24 & 22 & -2.94 \\
\hline Parietal_Inf_L & -63 & -9 & 30 & 17 & 2.76 \\
\hline Precentral_L & -36 & -60 & 48 & 22 & 3.66 \\
\hline Supp_Motor_Area_R & -33 & -3 & 57 & 72 & -4.42 \\
\hline Supp_Motor_Area_L & 6 & -18 & 72 & 150 & -3.72 \\
\hline
\end{tabular}

(d)

Fig. 2 Images of fALFF in the transverse plane (a), coronal plane (b), and sagittal plane (c) to show areas that had significantly different activity intensity between the Group-PDN and Group-N. Two-independent samples $t$ test was performed. Red color indicates an area that has an increased activity intensity of the Group PDN, and blue indicates a decrease. (d) Quantification of peak region

depression scores, eGFR, HbA1c level, HOMA-IR and HOMA- $\beta$ ( $p>0.05$; Fig. 5).

\section{DISCUSSION}

The main results of our study are as follows: (1) patients with PDN had increased insulin resistance, depression and anxiety than did patients with non-painful neuropathy; (2) multiple from the $x, y$ and $z$ coordinates. Peak region obtained using the automated anatomical labeling (AAL) function; $x, y$, $z$ coordinates of primary peak locations in the Montreal Neurological Institute (MNI) space. $K$ value represents the continuous voxel value; positive $t$ value indicates the fALFF value increases; negative $t$ value indicates the fALFF decreases

brain regions in patients with diabetic neuropathy exhibited abnormities in terms of neuron activity and synchrony; (3) compared with T2D patients with non-painful neuropathy, those with PDN showed abnormal activities in several brain regions, including the somatosensory, cognitive, emotional and other related brain regions; (4) the disease duration of diabetes, HbA1c level, HOMA-IR values and 


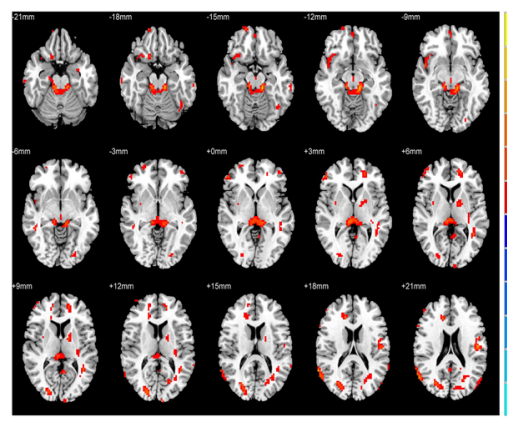

(a)

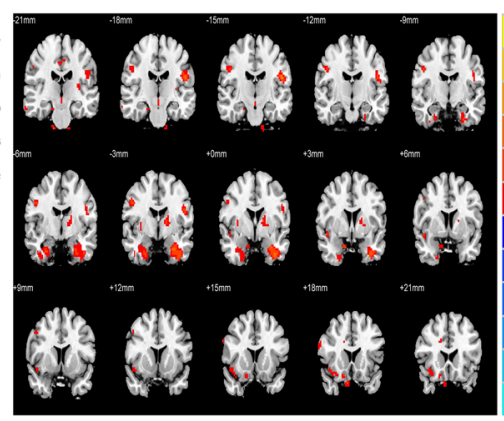

(b)

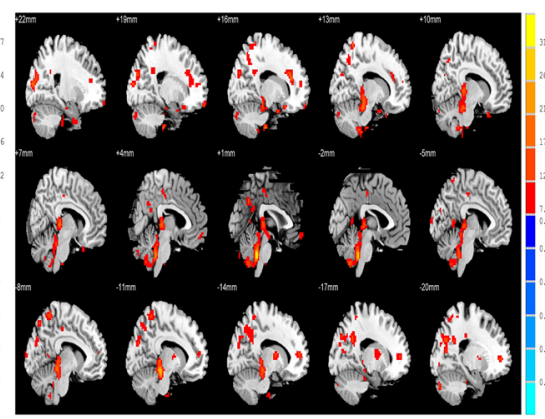

(c)
Fig. 3 Images of regional homo- geneity ( $\mathrm{ReHo}$ ) in the transverse plane (a), coronal plane (b), and sagittal plane (c) to show areas that had significantly different activity synchronism among the three groups. The ReHo data was analyzed by analysis of variance (ANOVA). Red color indicates an area that has significant difference in activity synchronism

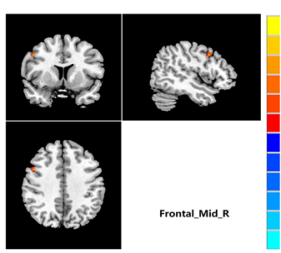

(a)

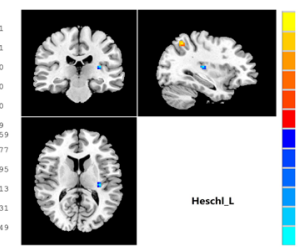

(b)

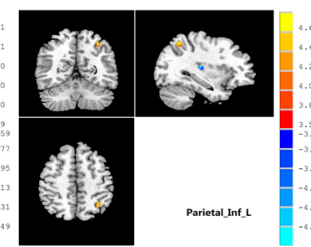

(c)

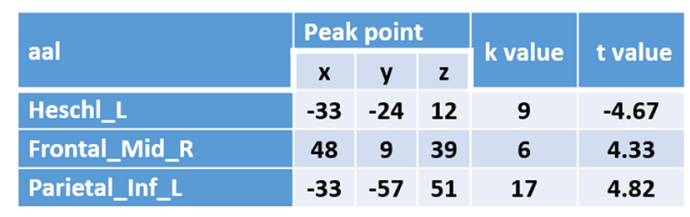

(d)
Fig. 4 Images of ReHo in the Frontal_Mid_R (a), Heschl_L (b) and Parietal_Inf_L (c) sequences to show areas that had increased activity synchronism. Twoindependent samples $t$ test was performed between the Group PDN and Group N. Red color indicates an area that has increased activity synchronism in the Group PDN, and blue indicates a decrease. (d) Quantification of

eGFR values were significantly correlated to abnormal spontaneous activity in some brain regions.

PDN is a type of neuropathic pain experienced by patients with diabetes. It can lead to mental problems, such as anxiety and depression [2]. Present-day treatment options for PDN are far from satisfactory, primarily because the pathophysiological mechanism remains known [2]. Tesfaye et al. reported that PDN is not only a disease of the peripheral nervous system but that it also involves the entire pain transmission pathway, including the central nervous system [3].

The rapid development of MRI as a diagnostic tool has facilitated knowledge of the pathophysiological mechanism of the brain $x, y$ and $z$ coordinates of primary peak locations in the Montreal Neurological Institute (MNI) space. Peak region obtained from AAL; $x, y z$ coordinates of primary peak locations in the MNI space. $K$ value represented the continuous voxel value; positive $t$ value indicates the ReHo value increases; negative $t$ value indicates the ReHo value decreases

structure and function in relation to many diseases, including PDN $[3,10,11]$. Rs-fMRI values can reflect the strength and connectivity of neural activity in pain circuits, thus detecting the occurrence and conduction of pain in these patients. In the present study, we found significant differences in the activity of multiple brain regions between patients with diabetic neuropathy and healthy controls, suggesting that diabetes and neuropathy are closely related to abnormal functioning of the brain. Interestingly, our data showed that patients with PDN had severe insulin resistance compared to those without PDN or healthy control subjects. Based on this result, we recommend that patients with severe insulin resistance should monitored for the development of PDN. 


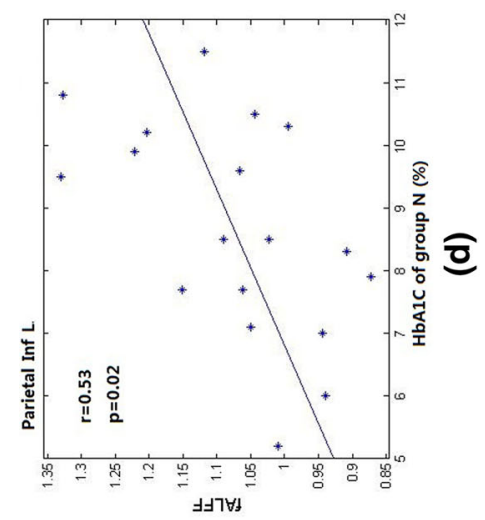

돈 욤듕 둗

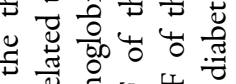

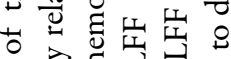

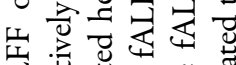

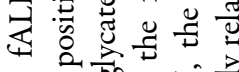

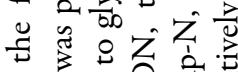

z

인

它 要品心

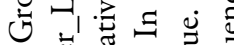
s 5 ơ

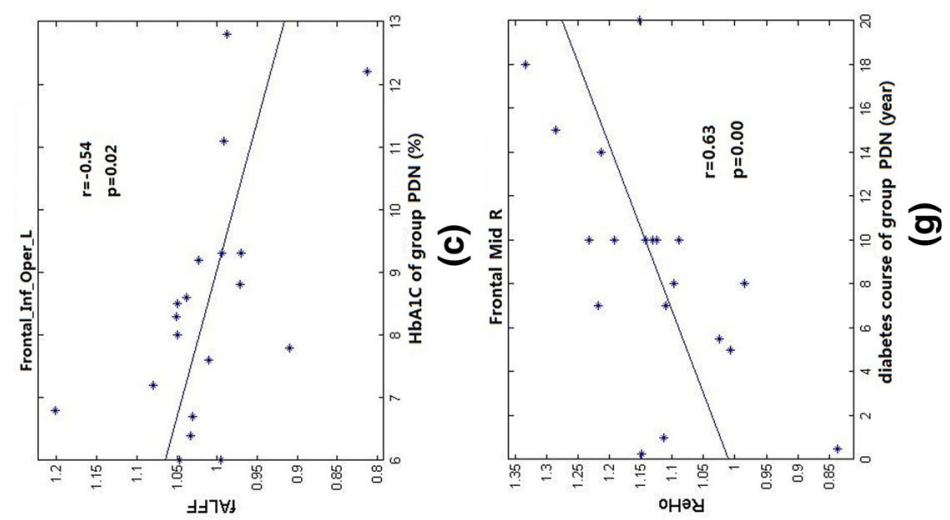

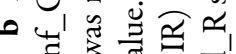

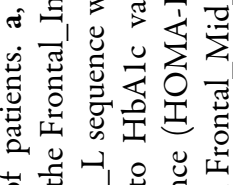

으

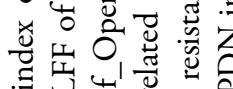

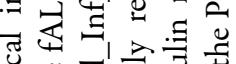

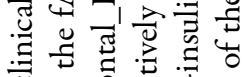

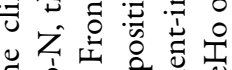

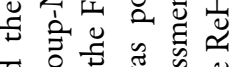

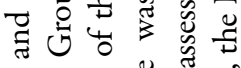
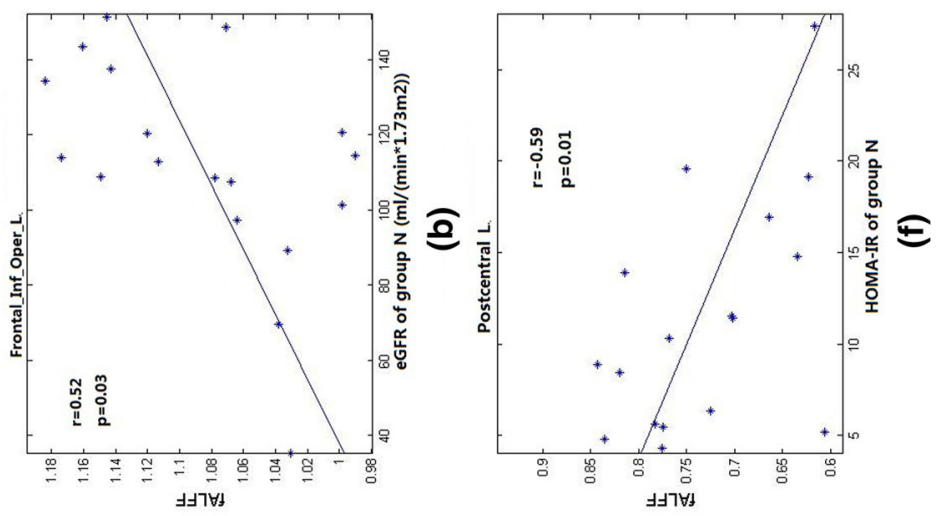

气.崖芯艺

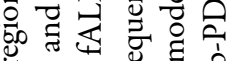

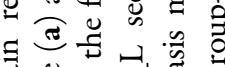

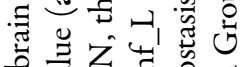

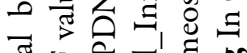

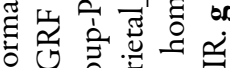

Є

त 00

$\Xi$ 퐁ํㅇ

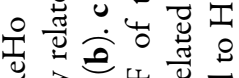

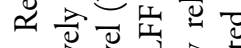

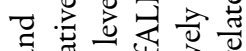

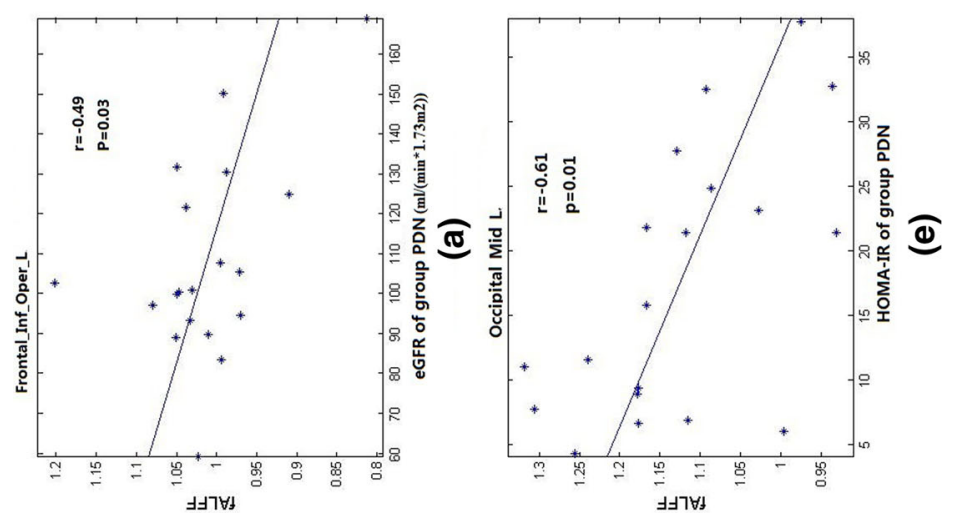

包现

茨要

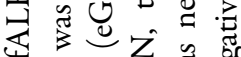

=

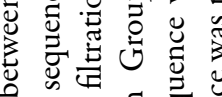

可豆

苛芯芯

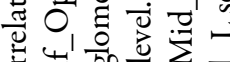

ن

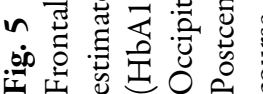


Our results also showed that there were differences in the fALFF between the Group-PDN and and Group-N and that these differences were mainly located in the temporal lobe, occipital lobe, parietal lobe, anterior central gyrus, central posterior gyrus and the supplementary motor area. These areas are mainly related to somatosensory functions, cognition and emotion [12]. The temporal lobe is related to memory, hearing, emotion and mental activity, and the occipital lobe is mainly responsible for visual processing. Patients with diabetic neuropathy often suffer from retinopathy and impaired vision, indicating that the brain area associated to visual functions is dysfunctional. The parietal lobe is involved in sensory processing and episodic memory retrieval [13]. The posterior cortex is an important part of the cerebral cortex and limbic system pathway, and it may participate in the learning and memory of pain [14]. The postcentral gyrus is mainly responsible for the acceptance and integration of sensation; and patients with PDN suffer from limb hyperalgesia and the symptoms of temperature loss.

The difference in ReHo between Group-PDN and Group-N was mainly found in the Frontal_Mid_R, Parietal_Inf_L and Heschl_L sequences. Heschl is the auditory center. The synchronization of PDN patients in this area is weakened, suggesting that the auditory network is damaged. The frontal lobe is an important part of the default mode network (DMN), and it plays an important role in cognition and emotion. The brain network activity of the DMN is more active when it is quiet and restful, inferring that patients with PDN are in more pain during night rest than during daily activities.

Pain is transmitted as stimulation of the sensory fibers through the spinal dorsal horn to the thalamus and then to different parts of the cerebral cortex. Sewards and Sewards [15] suggested that pain is mediated by multiple pathways, including through the transmission of sensory information in the thalamus and sensory cortex and the transmission of emotional information in such brain regions as the limbic system. In other words, the production of pain is regulated by two pathways: lateral perception recognition and medial emotional cognition
[16]. The results of our study suggest that there were also abnormalities in the sensory and emotional brain regions and their associated brain regions in our patients with PDN. Li et al. reported that patients with diabetic neuropathy not only show abnormalities in the brain region of the somatosensory pathway (insula, caudate nucleus, frontal lobe, cingulate gyrus), but also in brain regions related to cognition (temporal lobe, sea horse, spindle gyrus) [17]. Our results are consistent with this study.

The correlation analysis we conducted showed that the ReHo and fALFF values of abnormal activity brain regions in Group-PDN and Group-N were significantly correlated with the course of diabetes, eGFR, HbA1c levels and HOMA-IR values. We therefore logically speculate that diabetes may further lead to the development of neuropathy. We did not observe significant correlations between the rs-fMRI results and the anxiety and depression scores of the patients in this study. One possible explanation for this lack of significant correlation may be that the patients in this study had not reached a sufficiently severe degree of anxiety and depression to be measured and that the neuropathy had not significantly affected the activity of the brain.

This study has a number of limitations. Firstly, there are sampling errors because of the small sample size, so we cannot draw a solid conclusion from our data; further studies are needed with a larger sample size to verify these results. Secondly, this was a cross-sectional study and as such we draw a causal relationship between abnormal activity of the brain and the occurrence of the disease; a prospective longitudinal study is needed for further clarification. Thirdly, the neuropsychiatric scale lacks diabetes-related cognitive memory function assessment; however, it is necessary to link cognitive situation with the brain function. Fourthly, a simple diabetic control group without neuropathy was needed.

\section{CONCLUSION}

In conclusion, patients with PDN have abnormal neural activity in the somatosensory, cognitive and emotional regions of the brain. Our 
data may provide new information pertaining to further elucidation of the mechanism of PDN.

\section{ACKNOWLEDGEMENTS}

We thank the participants of the study.

Funding. This study was supported by grants from Nanjing Science and Technology Development Project (201605027). The article processing charges were funded by the authors.

Authorship. All named authors meet the International Committee of Medical Journal Editors (ICMJE) criteria for authorship for this article, take responsibility for the integrity of the work as a whole, and have given their approval for this version to be published.

Disclosures. Qing Zhang, Peng Zhang, Rui Yan, Xiang-hong Xu, Cun-nan Mao, Xiao-mei Liu, Feng-fei Li, Jian-hua Ma, Lei Ye, Zhi-jian Yao and Jin-dan $\mathrm{Wu}$ have nothing to disclose.

Complaince with Ethics Guidelines. All procedures performed in studies involving human participants were in accordance with [The First hospital of Nanjing, Nanjing, China] and with the 1964 Helsinki declaration and its later amendments or comparable ethical standards.

Informed Consent. Informed consent was obtained from all individual participants included in the study.

Data Availability. All data generated or analyzed during this study are included in this published article.

Open Access. This article is distributed under the terms of the Creative Commons Attribution-NonCommercial 4.0 International License (http://creativecommons.org/licenses/ by-nc/4.0/), which permits any noncommercial use, distribution, and reproduction in any medium, provided you give appropriate credit to the original author(s) and the source, provide a link to the Creative Commons license, and indicate if changes were made.

\section{REFERENCES}

1. Abbott CA, Malik RA, Van RERE, Jai K, Boulton AJM. Prevalence and characteristics of painful diabetic neuropathy in a large community-based diabetic population in the UK. Diabetes Care. 2011;34(10):2220-4.

2. Dobrota VD, Hrabac P, Skegro D, Smiljanic R, Dobrota S, Prkacin I, Brkljacic N, Peros K, Tomic M, Lukinovic-Skudar V, Kes VB. The impact of neuropathic pain and other comorbidities on the quality of life in patients with diabetes. Health Qual Life Outcomes. 2014;12:171.

3. Tesfaye S, Selvarajah D, Gandhi R, Greig M, Shillo P, Fang F, Wilkinson ID. Diabetic peripheral neuropathy may not be as its name suggests: evidence from magnetic resonance imaging. Pain. 2016;157[Suppl 1]:S72-80.

4. Jensen TS, Backonja MM, Jiménez SH, Tesfaye S, Valensi P, Ziegler D. New perspectives on the management of diabetic peripheral neuropathic pain. Diabetes Vasc Dis Res. 2006;3(2):108-19.

5. Pop-Busui R, Boulton AJ, Feldman EL, Bril V, Freeman R, Malik RA, Sosenko JM, Ziegler D. Diabetic neuropathy: a position statement by the American Diabetes Association. Diabetes Care. 2017;40(1):136-54.

6. Wager TD, Atlas LY, Lindquist MA, Roy M, Woo CW, Kross E. An fMRI-based neurologic signature of physical pain. N Engl J Med. 2013;368(15):1388-97.

7. Cui Y, Jiao Y, Chen YC, Wang K, Gao B, Wen S, Ju S, Teng G-J. Altered spontaneous brain activity in type 2 diabetes: a resting-state functional MRI study. Diabetes. 2014;63(2):749-60.

8. Selvarajah D, Wilkinson ID, Maxwell M, Davies J, Sankar A, Boland E, Gandhi R, Tracey I, Tesfaye S. Magnetic resonance neuroimaging study of brain structural differences in diabetic peripheral neuropathy. Diabetes Care. 2014;37(6):1681-8.

9. Meng J, Ge J, Zhang K, Yu C, Xu L, Gu Q, Jia W, Fang J, Hu X, Li J. Relationship between bone mineral density and $\mathrm{C}$ peptide level in male type 2 diabetes patients. Chin J Osteoporos. 2017;23(1):47-50 (in Chinese).

10. Cauda F, Sacco K, D'Agata F, Duca S, Cocito D, Geminiani G, Migliorati F, Isoardo G. Low- 
frequency BOLD fluctuations demonstrate altered thalamocortical connectivity in diabetic neuropathic pain. BMC Neurosci. 2009;10:138.

11. Cauda F, Sacco K, Duca S, Cocito D, D'Agata F, Geminiani GC, Canavero S. Altered resting state in diabetic neuropathic pain. PLoS One. 2009;4(2):e4542.

12. Jain R, Jain S, Raison CL, Maletic V. Painful diabetic neuropathy is more than pain alone: examining the role of anxiety and depression as mediators and complicators. Curr Diabetes Rep. 2011;11(4):275-84.

13. Raichle ME, MacLeod AM, Snyder AZ, Powers WJ, Gusnard DA, Shulman GL. A default mode of brain function. Proc Natl Acad Sci USA. 2001;98(2):676-82.

14. Sehlmeyer C, Schöning S, Zwitserlood P, Pfleiderer B, Kircher T, Arolt V, Konrad C. Human fear conditioning and extinction in neuroimaging: a systematic review. PLoS One. 2009;4(6):e5865.

15. Sewards TV, Sewards M. Separate, parallel sensory and hedonic pathways in the mammalian somatosensory system. Brain Res Bull. 2002;58(3):243-60.

16. Wu B, Fa Z, Lai C, Guo S, Guo Y. Abnormal activity of brain detected by functional magnetic resonance imaging in a rat model of diabetic neuropathic pain. Chin J Clin Anat. 2016;34(4):407-11 (in Chinese).

17. Li J, Zhang $\mathrm{W}$, Wang $\mathrm{X}$, Yuan $\mathrm{T}$, Liu $\mathrm{P}$, Wang $\mathrm{T}$, Shen L, Huang Y, Li N, You H, Xiao T, Feng F, Ma C. Functional magnetic resonance imaging reveals differences in brain activation in response to thermal stimuli in diabetic patients with and without diabetic peripheral neuropathy. PLoS One. 2018;13(1):e0190699. 